\title{
Robust Spin Polarization and Spin Textures on Stepped Au(111) Surfaces
}

\author{
Jorge Lobo-Checa, ${ }^{1,2,3}$ Fabian Meier, ${ }^{2,3}$ Jan Hugo Dil, ${ }^{2,3}$ Taichi Okuda, ${ }^{2,4}$ Martina Corso, ${ }^{2}$ Vladimir N. Petrov, ${ }^{5}$ \\ Matthias Hengsberger, ${ }^{2}$ Luc Patthey, ${ }^{3}$ and Jürg Osterwalder ${ }^{2}$ \\ ${ }^{1}$ Centre d'Investigaciò en Nanociència i Nanotecnologia, CIN2 (CSIC-ICN), Esfera UAB, Campus de Bellaterra, \\ 08193-Barcelona, Spain \\ ${ }^{2}$ Physik-Institut, Universität Zurich-Irchel, Winterthurerstrasse 190, CH-8057 Zürich, Switzerland \\ ${ }^{3}$ Swiss Light Source, Paul Scherrer Institut, CH-5232 Villigen, Switzerland \\ ${ }^{4}$ The Institute for Solid State Physics, The University of Tokyo, Kashiwanoha 5-1-5, Kashiwa 277-8581, Japan \\ ${ }^{5}$ St. Petersburg Technical University, 29 Polytechnicheskaya Street, St. Petersburg 195251, Russia
}

(Received 3 December 2009; published 7 May 2010)

\begin{abstract}
The influence of structural defects, in the form of step lattices, on the spin polarization of the spin-orbit split Shockley surface state of $\mathrm{Au}(111)$ has been investigated. Spin- and angle-resolved photoemission data from three vicinal surfaces with different step densities are presented. The spin splitting is preserved in all three cases, and there is no reduction of the spin polarization of individual subbands, including the umklapp bands induced by the step lattice. On the sample with the highest step density studied, where the wave functions are delocalized over several terraces, the spin splitting is enhanced substantially, likely as an effect of the effective surface corrugation as on related surface alloys. The spin texture shows in all cases spin polarization vectors tangential to the Fermi circles, with the same helicities as on $\mathrm{Au}(111)$.
\end{abstract}

PACS numbers: 79.60.Bm, 72.25.-b, 73.20.At

The Rashba effect is one of the cornerstones of spintronics [1]. It applies to an electron gas confined to two dimensions (2D) by an asymmetric potential and leads to a momentum-dependent spin splitting of the electronic states, induced by the spin-orbit interaction [2]. The splitting can be controlled by applying an electric field along the confinement direction, thus providing effective control of injected spin currents. Such systems have been realized in semiconductor heterostructures [3], but they exist also naturally on noble metal surfaces [4], as has been shown directly by spin- and angle-resolved photoemission spectroscopy (SARPES) [5]. While these latter systems are obviously less suitable for device applications, they offer a convenient platform for fundamental studies of these spin-split states.

In this Letter we address the influence of lateral confinement and presence of structural defects on the spin polarization and on the spin splitting. Vicinal $\mathrm{Au}(111)$ surfaces are used as model systems, because the Shockley surface state on $\mathrm{Au}(111)$ shows the largest spin-orbit splitting of the noble metal surfaces, with $100 \%$ spin polarized subbands. Such vicinals exhibit regular lattices of monoatomic steps, and flat terraces in between [6]. Linear steps represent one kind of structural defects; they act as repulsive potential barriers for surface state electrons that can lead to partial or even complete lateral confinement of the surface state wave function, depending on the terrace width [7]. According to Bihlmayer et al., a large contribution to the spin splitting on $\mathrm{Au}(111)$ originates from the topmost atomic layer [8], and it should thus be susceptible to the presence of steps.

On the flat $\mathrm{Au}(111)$ surface, the parabolic subbands are shifted in momentum by $0.026 \AA^{-1}$ with respect to each other $[4,5,9]$. The resulting momentum distribution close to the Fermi level thus consists of two concentric rings, with spin polarization vectors lying mainly in-plane and tangential to the circular shape [4,5]. Interestingly, the 2D Fourier analysis of surface state standing wave patterns, originating from defect scattering and imaged by scanning tunneling microscopy (STM), produces only a single circle at the Fermi energy [10]. This apparent inconsistency was explained by introducing an additional spin selection rule, allowing for interference effects only between bands with parallel spin polarization $[11,12]$. While this represented a nice confirmation of the spin texture of these states in momentum space, a direct investigation of the interaction of these states and their spin polarization with surface defects can only be achieved by SARPES. Indeed, a (spin-integrated) angle-resolved photoemission (ARPES) investigation of some vicinal $\mathrm{Au}(111)$ surfaces has shown that the spin splitting persists for surfaces with large terraces [13].

The effects of a regular array of steps on the $\mathrm{Au}(111)$ Shockley state are fourfold: First, the energy of the band bottom shifts towards the Fermi energy because of the repulsive scattering potential $[14,15]$. Second, a momentum shift of the band bottom occurs in the direction perpendicular to the steps. Its magnitude is related to both the complex potential landscape created by the steps and the existence of a projected gap at the $\bar{\Gamma}$ point [14]. Qualitatively, this effect is explained as a change in the reference of the electron wave function: For large separation between steps the electrons are confined within each (111) terrace [Fig. 1(a)] and openings of small energy gaps have been observed [13-15]. For small step separation the electrons interact with several steps. Their wave functions 
are therefore delocalized and sensitive to the step lattice [Fig. 1(b)]. This transition is accompanied by the closure of the surface-projected bulk energy gap at the $\bar{\Gamma}$ point, transforming the Shockley surface state into a surface resonance $[16,17]$. Third, the step superlattice also generates umklapp states, i.e., replica bands shifted in momentum in the direction perpendicular to the steps by $2 \pi / d$ [Fig. 1(c)] [13-15]. Finally, the spin-orbit induced splitting of the subbands is progressively lost in the ARPES spectra due to the peak broadening resulting from the large terrace width distributions (TWD). This splitting can only be observed with optimally prepared surfaces, at low temperatures, and only for the largest terrace widths of the order of $\sim 50 \AA$ [13].

The vicinal surfaces studied in this work are $\operatorname{Au}\left(1112\right.$ 12), $\operatorname{Au}\left(7 \quad 8\right.$ 8) and $\operatorname{Au}\left(\begin{array}{lll}2 & 2 & 3\end{array}\right)$. The relevant structural parameters are introduced in Fig. 1(d) and summarized in Fig. 2. $\mathrm{Au}\left(\begin{array}{ll}11 & 12 \\ 12\end{array}\right)$ and $\mathrm{Au}(7 \mathrm{8} 8)$ have large terraces and show surface reconstructions similar to $\operatorname{Au}(111)$, whereas $\operatorname{Au}\left(\begin{array}{lll}2 & 2 & 3\end{array}\right)$ exhibits small terraces, so that the aforementioned localization transition occurs in this set. All surfaces were prepared in situ in ultrahigh vacuum (UHV) by repeated cycles of $\mathrm{Ar}^{+}$sputtering at $0.75 \mathrm{kV}$ and annealing to $700 \mathrm{~K}$. The quality of the surfaces was checked from the spot splitting in low-energy electron diffraction (LEED) patterns and the line shape and width of the surface state in spin-integrated ARPES. No minigaps were observed in the surface state dispersion due to a combination of TWD, acquisition temperature and insufficient instrumental resolution [6]. Spin-integrated and spin- a)
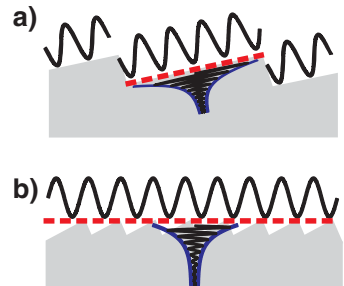

c)

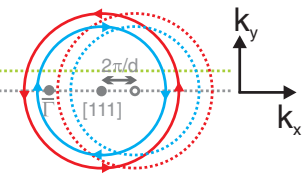

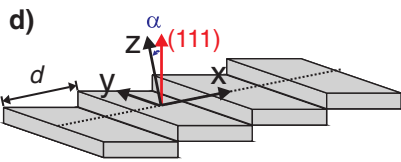

e)

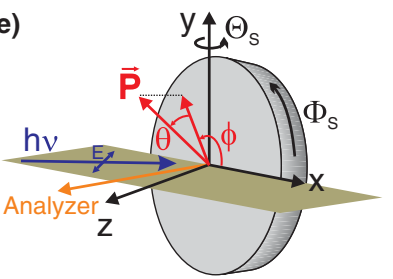

FIG. 1 (color online). Surface state wave functions for two extreme cases of step densities on vicinal $\mathrm{Au}(111)$ : (a) the electrons are confined within each terrace and (b) they are delocalized on the average surface. (c) Graphical representation, for case (a), of the main Fermi surfaces (solid circles) and the umklapp states (dotted circles) induced by the step superlattice. Directions of the tangential in-plane spin polarization vectors are included. The sample coordinate system and the experimental setup are schematically represented in (d) and (e), respectively. $\alpha$ is the miscut angle and $d$ is the distance between steps. A slight sample tilt around the $x$-axis could not be corrected, therefore momentum scans along $k_{x}$ follow the green dashed line in (c) rather than the high symmetry gray line. resolved ARPES data were taken at the COPHEE end station [18] located at the Surface and Interface Spectroscopy (SIS) beam line at the Swiss Light Source. All measurements were performed at room temperature (RT) in a UHV chamber with $1 \times 10^{-10}$ mbar base pressure using linear horizontal polarized light. The energy and angular resolution of our SARPES measurements were $70 \mathrm{meV}$ and $\pm 0.5^{\circ}$ at $21 \mathrm{eV}$ photon energy. Spin-resolved momentum distribution curves (MDCs) were obtained by recording intensities and scattering asymmetries in two orthogonal Mott detectors [18] for different emission angles.

Figure 2 summarizes the spin-integrated Fermi surface maps and band dispersion measurements perpendicular to the steps for the three vicinal surfaces, showing the parabolic dispersion of the Shockley surface state. Although similar data were already reported in literature $[6,13,14]$, they are included here to serve as a guideline for the discussion of the spin-resolved data. No spin splitting can be resolved in these data because the bands are intrinsically broad. This is a consequence of the large TWD in these samples, and of the measurement temperature (RT). Weak replica bands, shifted to larger $k_{x}$ values, are observed for $\mathrm{Au}(111212)$ and $\mathrm{Au}(788)$, both in the energy distribution curves (EDCs) and Fermi surface maps (FSMs). They are due to umklapp scattering within the step lattice. It is not observed for $\operatorname{Au}(223)$, because the displayed momentum range is too small (the center of the replica is found at $k_{x}=$ $\left.0.79 \AA^{-1}[19]\right)$.

The evolution of the spin polarization and spin texture of the surface state with increasing step density is investigated through spin-resolved MDCs measured along $k_{x}$, i.e., the direction perpendicular to the steps (Fig. 3). Two orthogonal Mott polarimeters yield simultaneously the spin-

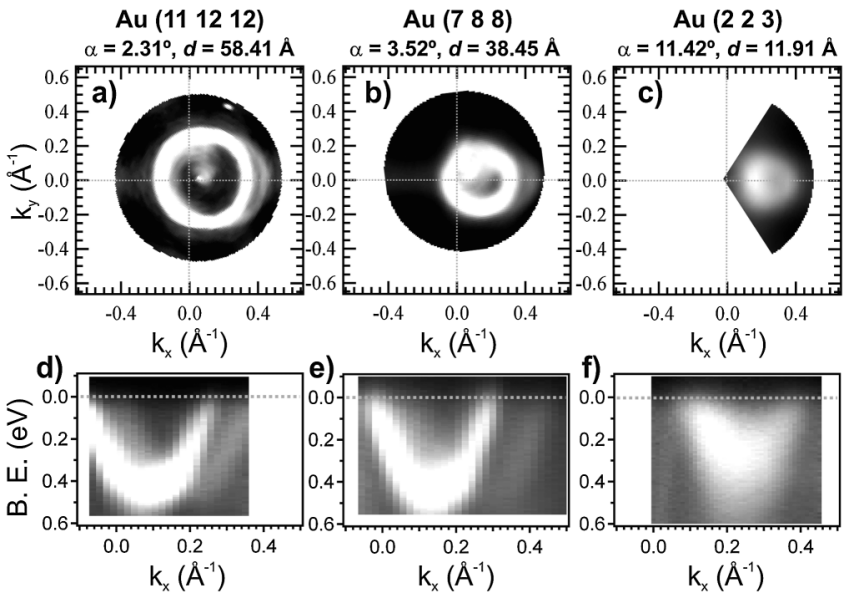

FIG. 2. Spin-integrated ARPES data from Au(11 12 12), $\operatorname{Au}\left(\begin{array}{lll}7 & 8 & 8\end{array}\right)$ and $\operatorname{Au}\left(\begin{array}{lll}2 & 2 & 3\end{array}\right)$, representing Fermi surface maps (a)-(c) and band dispersions perpendicular to the steps (d)-(f), taken with $21 \mathrm{eV}$ photon energy. The surface state bands are broadened due to the terrace width distribution, completely masking the spin splitting. For $\mathrm{Au}\left(\begin{array}{lll}11 & 12 & 12\end{array}\right)$ and $\mathrm{Au}\left(\begin{array}{l}7 \\ 8\end{array}\right.$ weak replica due to umklapp scattering are observed. 

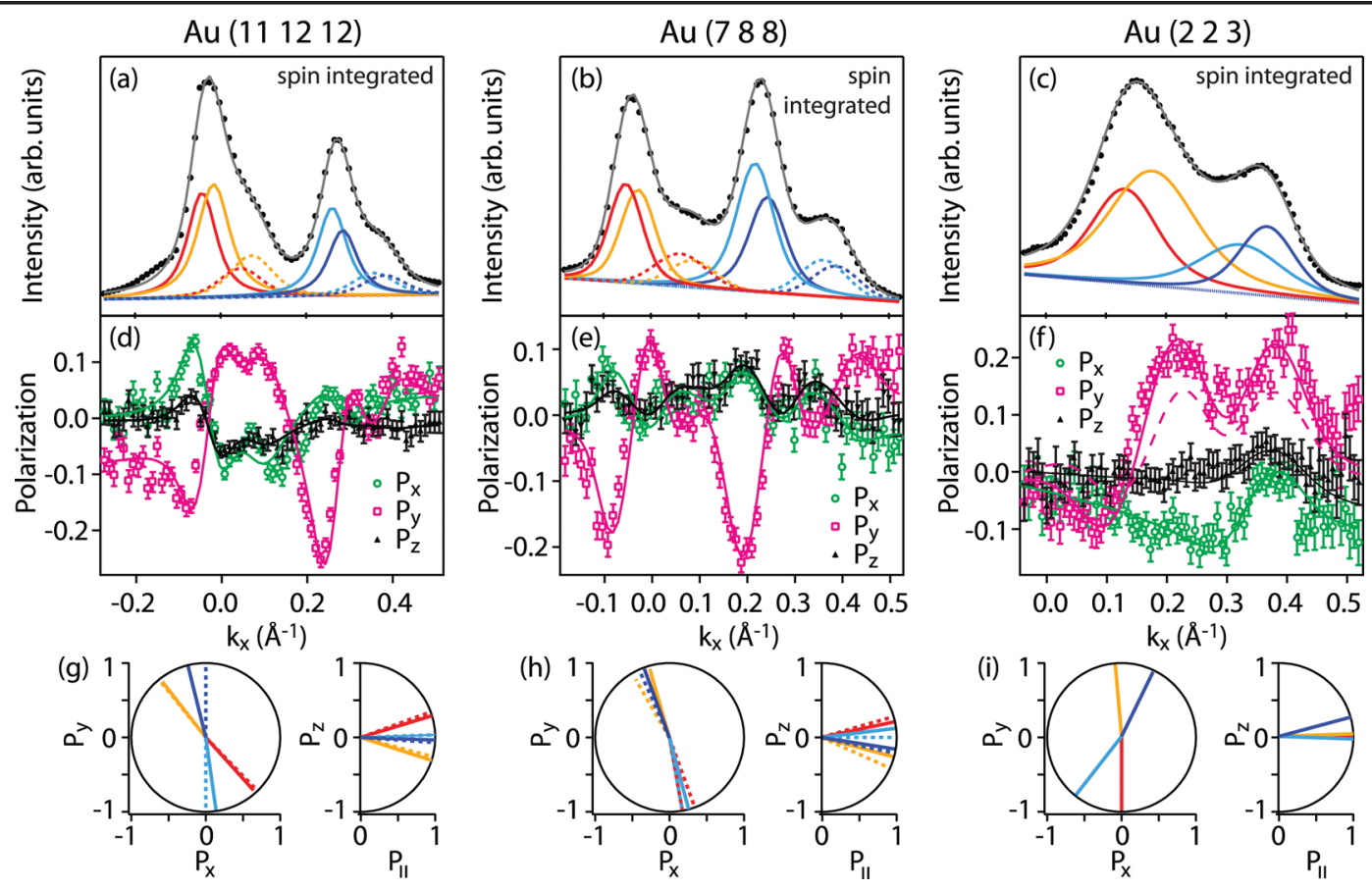

FIG. 3 (color). Spin-resolved MDCs from the three investigated vicinal surfaces taken with 23 eV photon energy [binding energies: $0.10 \mathrm{eV}$ for $\mathrm{Au}\left(1112\right.$ 12) and $\mathrm{Au}(788), 0.05 \mathrm{eV}$ for $\mathrm{Au}(223)$ ] obtained by rotation of $\Theta_{s}$ in Fig. 1(e). The top graphs (a)-(c) give the spin-integrated intensities, showing also the fitted peak profiles used for the two-step fitting routine described in the text. In the graphs (d)-(f), the corresponding spin polarization curves and errors for the $x$ (green open circles), $y$ (magenta open squares), and $z$ (black triangles) components are represented. The polarization fits (see text) are shown as continuous lines in the corresponding color. The discontinuous line in (f) corresponds to the $y$ polarization fit when fixing the splitting to the flat Au(111) value. The bottom panels (g)(i) show the resulting polarization vectors for the individual peaks. In the top and bottom graphs the components from the main (umklapp) states are indicated as continuous (discontinuous) lines using the same color codes. Each of the bottom panels gives the inplane (left) and the out-of-plane (right) orientation of the spin polarization vectors.

integrated intensities and the three spin polarization components $P_{x}, P_{y}$ and $P_{z}$ [18]. In order to quantify the relative contributions of all the sub-bands the analysis of these data was based on a two-step fitting procedure described in detail elsewhere [20,21]. For these fits four pairs of Voigt peaks were used for $\operatorname{Au}\left(\begin{array}{lll}11 & 12 & 12\end{array}\right)$ and $\operatorname{Au}\left(\begin{array}{l}7 \\ 8\end{array}\right.$ 8) (the umklapp peaks are interweaved with the main peaks) and only two pairs for $\mathrm{Au}\left(\begin{array}{l}2 \\ 2\end{array} 3\right)$ on a linear background (see Ref. [19] for details). The use of pairs of peaks is required due to the strong modulation observed in the spin polarization data. In order to comply with the Rashba model [2], the spin splittings were forced to be equal for all peak pairs. This analysis yields the value of the splitting, the degree of spin polarization of each peak, and the orientations of the polarization vectors, which are summarized for each surface in Figs. 3(g) to 3(i). The splittings $\Delta k$ and the peak widths are listed in Table I. The widths of all main peaks are quite similar for a particular surface, and average values are therefore given. The same holds for umklapp peaks.

Several conclusions can be drawn from these data and their fits. (i) The surface states show clear spin polarization signals for all three surfaces. (ii) Excellent fits could be obtained when setting all values $c_{i}=1.0$; i.e., all peaks appear to be $100 \%$ spin polarized. (iii) All spin polarization vectors lie mainly in-plane as in the case of the flat (111) surface. All paired peaks have opposite polarization vectors. (iv) The spin polarization vectors of the umklapp states follow closely, in direction and magnitude, those of the main states. (v) The widths of the components increase with decreasing terrace size, as previously observed and related to an increase of the TWD [14,15]. The umklapp peaks are broader than the main peaks but follow the same tendency. (vi) $\operatorname{Au}\left(\begin{array}{lll}11 & 12 & 12\end{array}\right)$ and $\operatorname{Au}(788)$ show the same spin splitting $\Delta k$ as $\mathrm{Au}(111)\left(0.026 \AA^{-1}\right)$. On the other hand, $\operatorname{Au}(223)$ shows a substantially larger splitting of $0.042 \AA^{-1}$.

TABLE I. Spin splittings, expressed as momentum shift $\Delta k$ (in $\AA^{-1}$ ), and average peak widths (in $\AA^{-1}$ ) obtained from the fits to the data of Fig. 3 [binding energies: $0.10 \mathrm{eV}$ for $\mathrm{Au}\left(\begin{array}{lll}11 & 12 & 12\end{array}\right)$ and $\mathrm{Au}\left(7 \mathrm{8}\right.$ 8), $0.05 \mathrm{eV}$ for $\mathrm{Au}\left(22_{2} 3\right)$ ]. $\mathrm{Au}(111)$ is added for completeness.

\begin{tabular}{lcccc}
\hline \hline & \multicolumn{2}{c}{ Main state } & \multicolumn{2}{c}{ Umklapp state } \\
\multicolumn{1}{c}{ Surface } & $\Delta k$ & FWHM & $\Delta k$ & FWHM \\
\hline $\mathrm{Au}\left(\begin{array}{lll}1 & 1 & 1\end{array}\right)$ & 0.026 & 0.053 & & \\
$\mathrm{Au}\left(\begin{array}{llll}1 & 1 & 12 & 12\end{array}\right)$ & 0.026 & 0.080 & 0.026 & 0.101 \\
$\mathrm{Au}\left(\begin{array}{lll}7 & 8 & 8\end{array}\right)$ & 0.026 & 0.098 & 0.026 & 0.112 \\
$\mathrm{Au}\left(\begin{array}{llll}2 & 2 & 3\end{array}\right)$ & 0.042 & 0.146 & & \\
\hline \hline
\end{tabular}


The fact that the surface state electrons remain fully spin polarized on these three stepped surfaces merits further discussion. It indicates a high robustness of spin currents in the presence of such structural defects. Spin polarization is neither lost by scattering within the ordered step lattice nor by the high degree of disorder that is obvious by the large TWDs. Even umklapp bands preserve the full spin polarization. The close resemblance of all measured surfaces to the $\mathrm{Au}(111)$ case strongly suggests that the observed spin polarization vectors essentially reflect the initial state spin structure [22].

With regard to the spin texture, all vicinal surfaces show directions of spin polarization vectors similar to those on the flat $\mathrm{Au}(111)$ surface along the direction perpendicular to the steps $\left(k_{x}\right)$, i.e., the spin vectors point mainly along the tangential $\pm y$ axis [5], with the same helicities for the corresponding subbands. Umklapp bands closely follow their corresponding main bands. Deviations from a purely tangential in-plane spin texture appear as modulations in the measured $P_{x}$ curves. They are assigned to a small sample tilt of the order of $1^{\circ}$ that cannot be corrected in our experimental setup. Because of the small Fermi surface radii, it translates into a much larger rotation of the spin polarization vectors [Fig. 1(c)].

Finally, we discuss the strong enhancement of the spin splitting $\Delta k$ by $\sim 60 \%$ in the case of $\operatorname{Au}(223)$ with respect to the flat $\mathrm{Au}(111)$ and the other two investigated surfaces. This finding is completely hidden in the broad peak widths found on this surface and can only be revealed by a careful spin analysis. We have verified the validity of this result by imposing a splitting of $0.026 \AA^{-1}$, which is the splitting on $\mathrm{Au}(111)$, in a constrained fit. While the intensity fit is still excellent, the $P_{y}$ fit [shown as a dashed line in Fig. 3(f)] is much worse than the fit yielding $0.042 \AA^{-1}$ (continuous blue line). We propose that the enhanced spin splitting is related to the short terrace width and the concomitant change in reference frame for the electron wave function [see Fig. 1(b)]. In the case of $\mathrm{Au}\left(\begin{array}{lll}11 & 12 & 12\end{array}\right)$ and $\mathrm{Au}(7 \quad 8$ 8) the electrons are confined and referred to single (111) terraces, whereas for $\operatorname{Au}\left(\begin{array}{lll}2 & 2 & 3\end{array}\right)$ the electrons propagate with respect to the average macroscopic surface. Accordingly, the electrons interact with several steps, and their wave functions "feel" a strong increase of the effective surface corrugation on the densely stepped $\operatorname{Au}\left(\begin{array}{lll}2 & 2 & 3\end{array}\right)$ surface. In the case of surface alloys, it has been demonstrated that the enhanced potential gradient at the surface induced by the surface corrugation is responsible for the unexpected increase in the magnitude of the Rashba splitting [23-26]. Therefore, we speculate that the same mechanism applies for the vicinal $\mathrm{Au}(111)$ surfaces, but only above a critical step density leading to propagating wave functions. According to the literature, the transition occurs at terrace widths of $\sim 20 \AA$.

In summary, we have studied the interaction of spin polarized surface state electrons with a periodic distribution of steps by means of spin-resolved ARPES. We ob- serve the existence of spin splittings even when spinintegrated ARPES cannot resolve them. Moreover, our analysis shows that the spin polarization and the spin textures on vicinal surfaces are closely related to those on the flat $\mathrm{Au}(111)$ surface. All subbands, including umklapp bands due to the step lattice, are 100\% polarized and show essentially tangential spins and the original spin helicity of $\mathrm{Au}(111)$, in spite of a relatively high degree of disorder as reflected in the broad photoemission peaks. For the highest step density, we observe that the spin splitting increases substantially as the electron wave functions effectively probe an increased surface corrugation.

We wish to express our gratitude to J.E. Ortega and $\mathrm{R}$. Fasel for lending us the crystals studied in this work, to T. Greber for cunning and stimulating discussions, to $\mathrm{M}$. Klöckner for technical assistance, and to the staff of the Surface and Interface Spectroscopy (SIS) beam line of the Swiss Light Source (Paul Scherrer Institut, Switzerland), where these experiments were performed. This work has been supported by the Swiss National Science Foundation and by the Spanish Ramón y Cajal Program.

[1] S. A. Wolf et al., Science 294, 1488 (2001).

[2] Y. A. Bychkov and E. I. Rashba, JETP Lett. 39, 78 (1984).

[3] J. Nitta et al., Phys. Rev. Lett. 78, 1335 (1997).

[4] S. LaShell, B. A. McDougall, and E. Jensen, Phys. Rev. Lett. 77, 3419 (1996).

[5] M. Hoesch et al., Phys. Rev. B 69, 241401(R) (2004).

[6] M. Corso et al., J. Phys. Condens. Matter 21, 353001 (2009).

[7] J.E. Ortega et al., Phys. Rev. B 65, 165413 (2002).

[8] G. Bihlmayer et al., Surf. Sci. 600, 3888 (2006).

[9] F. Reinert et al., Phys. Rev. B 63, 115415 (2001).

[10] L. Petersen et al., Surf. Sci. 443, 154 (1999).

[11] L. Petersen et al., Surf. Sci. 459, 49 (2000).

[12] J. I. Pascual et al., Phys. Rev. Lett. 93, 196802 (2004).

[13] A. Mugarza et al., Phys. Rev. B 66, 245419 (2002).

[14] A. Mugarza and J. E. Ortega, J. Phys. Condens. Matter 15, S3281 (2003).

[15] F. Baumberger et al., Phys. Rev. Lett. 92, 196805 (2004).

[16] J. E. Ortega et al., Phys. Rev. Lett. 84, 6110 (2000).

[17] J. E. Ortega et al., Surf. Sci. 482-485, 764 (2001).

[18] M. Hoesch et al., J. Electron Spectrosc. Relat. Phenom. 124, 263 (2002).

[19] See supplementary material at http://link.aps.org/ supplemental/10.1103/PhysRevLett.104.187602 for more information on ARPES data, the SARPES fit procedure, and error analysis.

[20] F. Meier et al., Phys. Rev. B 77, 165431 (2008).

[21] F. Meier, J. H. Dil, and J. Osterwalder, New J. Phys. 11, 125008 (2009).

[22] J. Henk et al., J. Phys. Condens. Matter 16, 7581 (2004).

[23] K. Sugawara et al., Phys. Rev. Lett. 96, 046411 (2006).

[24] C. R. Ast et al., Phys. Rev. Lett. 98, 186807 (2007).

[25] G. Bihlmayer S. Blugel, and E. V. Chulkov, Phys. Rev. B 75, 195414 (2007).

[26] J. H. Dil, J. Phys. Condens. Matter 21, 403001 (2009). 